\title{
A|P| $\begin{aligned} & \text { Journal of } \\ & \text { Mathematical Physics }\end{aligned}$
}

Scalar field theory at finite temperature in $D=2+1$

G. N. J. Añaños

Citation: Journal of Mathematical Physics 47, 012301 (2006); doi: 10.1063/1.2159068

View online: http://dx.doi.org/10.1063/1.2159068

View Table of Contents: http://scitation.aip.org/content/aip/journal/jmp/47/1?ver=pdfcov

Published by the AIP Publishing

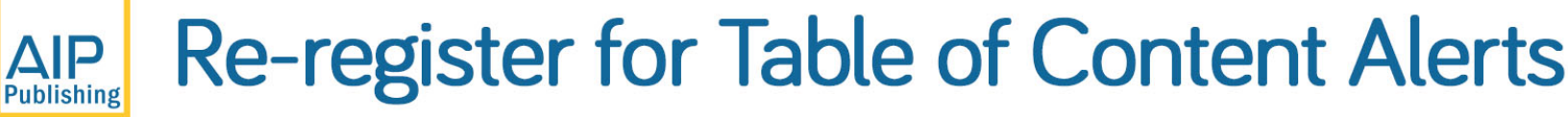




\title{
Scalar field theory at finite temperature in $D=2+1$
}

\author{
G. N. J. Añaños ${ }^{a)}$ \\ Instituto de Física Teórica-IFT, Universidade Estadual Paulista, Rua Pamplona 145, \\ São Paulo, SP 01405-900 Brazil
}

(Received 21 September 2005; accepted 8 November 2005;

published online 17 January 2006)

\begin{abstract}
We discuss the $\varphi^{6}$ theory defined in $D=2+1$-dimensional space-time and assume that the system is in equilibrium with a thermal bath at temperature $\beta^{-1}$. We use the $1 / N$ expansion and the method of the composite operator (Cornwall, Jackiw, and Tomboulis) for summing a large set of Feynman graphs. We demonstrate explicitly the Coleman-Mermin-Wagner theorem at finite temperature. (C) 2006 American Institute of Physics. [DOI: 10.1063/1.2159068]
\end{abstract}

\section{INTRODUCTION}

The conventional perturbation theory in the coupling constant or in $\hbar$, i.e., the loop expansion can only be used for the study of small quantum corrections to classical results. When discussing quantum mechanical effects to any given order in such an expansion, one is not usually able to justify the neglect of yet higher order. In other words, for theories with a large $N$ dimensional internal symmetry group, there exist another perturbation scheme, the $1 / N$ expansion, which circumvents this criticism. Each term in the $1 / N$ expansion contains an infinite subset of terms of the loop expansion. The $1 / N$ expansion has the nice property that the leading-order quantum corrections are of the same order as the classical quantities. Consequently, the leading order which adequately characterizes the theory in the large $N$ limit preserves much of the nonlinear structure of the full theory.

The scalar field $\left(\varphi^{4}\right)_{D=4}$ theory at finite temperature is of great interest in the field of phase transitions in the early universe and heavy ion collisions. When used as a simple model for the Higgs particle in the standard model of electroweak interactions, it may allow the study of symmetry breaking phase transitions in the early universe. For $N=4$ scalar fields, it is also a model of chiral symmetry breaking in QCD and hence is relevant for the theoretical study of heavy ion collisions. Moreover, this theory is an excellent theoretical laboratory in which analytic nonperturbative methods can be tested. Now for the case $D=3$ it has been shown that, in the large $N$ limit, the $\varphi^{6}$ theory has a UV fixed point and therefore must have a second IR fixed point ${ }^{1}$ and for this we could say that at least for large $N$ the $\left(\varphi^{6}\right)_{D=3}$ theory is known to be qualitatively different from $\left(\varphi^{4}\right)_{D=4}$ theory. For other ways, theories in less than four space-time dimensions can offer interesting and complex behavior as well as tractability, and, for example, the case of three space-time dimensions, they can even be directly physical, describing various planar condensed matter systems. For example, the introduction of the $\varphi^{6}$ term generates a rich phase diagram, with the possibility of second order, first order phase transitions or even both transitions occurring simultaneously. This situation defines the tricritical phenomenon. For example, some systems such antiferromagnets in the presence of a strong external field or the $\mathrm{He}^{3}-\mathrm{He}^{4}$ mixture exhibits such behavior.

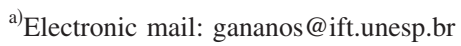




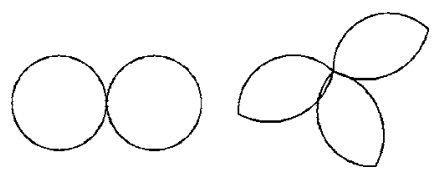

FIG. 1. The 2PI vacuum graphs.

\section{THE EFFECTIVE POTENTIAL}

The theory for which we are interested is given by the Lagrangian,

$$
\mathcal{L}(\varphi)=\frac{1}{2}\left(\partial_{\mu} \varphi\right)^{2}-\frac{1}{2} m_{0}^{2} \varphi^{2}-\frac{\lambda_{0}}{4 ! N} \varphi^{4}-\frac{\eta_{0}}{6 ! N^{2}} \varphi^{6}
$$

is a theory of $N$ real scalar fields with $O(N)$ symmetry.

For definiteness, we work at zero temperature; however, the finite temperature generalizations can be easily obtained. ${ }^{2}$

In this section we are going to use the method of composite operator developed by Cornwall, Jackiw, and Tomboulis (CJT) ${ }^{3,4}$ in order to get the effective potential $\Gamma(\phi)$ at leading order in the $1 / N$ expansion. The composite operator formalism reduces the problem to summing two particle irreducible (2PI) Feynman graphs by defining a generalized effective action $\Gamma(\phi, G)$ which is a functional not only of $\phi_{a}(x)$, but also of the expectation values $G_{a b}(x, y)$ of the time ordered product of quantum fields $\langle 0|T(\varphi(x) \varphi(y))| 0\rangle$, i.e.,

$$
\Gamma(\phi, G)=I(\phi)+\frac{i}{2} \operatorname{Tr} \operatorname{Ln} G^{-1}+\frac{i}{2} \operatorname{Tr} D^{-1}(\phi) G+\Gamma_{2}(\phi, G)+\cdots,
$$

where $I(\phi)=\int \mathrm{d} x^{D} \mathcal{L}(\phi), G$ and $D$ are matrices in both the functional and the internal space whose elements are $G_{a b}(x, y), D_{a b}(\phi ; x, y)$, respectively, and $D$ is defined by

$$
i D^{-1}=\frac{\delta^{2} I(\phi)}{\delta \phi(x) \delta \phi(y)} .
$$

The quantity $\Gamma_{2}(\phi, G)$ is computed as follows. In the classical action $I(\varphi)$ we must shift the field $\varphi$ by $\phi$. The new action $I(\varphi+\phi)$ possesses terms cubic and higher in $\varphi$. This defines an interaction part $I_{\text {int }}(\varphi, \phi)$ where the vertices depend on $\phi . \Gamma_{2}(\phi, G)$ is given by sum of all (2PI) vacuum graphs in a theory with vertices determined by $I_{\text {int }}(\varphi, \phi)$ and the propagators set equal to $G(x, y)$. The trace and logarithm in Eq. (2) are functional. After these procedures the interaction Lagrangian density becomes

$$
\begin{aligned}
\mathcal{L}_{\text {int }}(\varphi, \phi)= & -\frac{1}{2}\left(\frac{\lambda_{0} \phi_{a}}{3 N}+\frac{\eta_{0} \phi^{2} \phi_{a}}{30 N^{2}}\right) \varphi_{a} \varphi^{2}-\left(\frac{8 \eta_{0} \phi_{a} \phi_{b} \phi_{c}}{6 N^{2}}\right) \varphi_{a} \varphi_{b} \varphi_{c}-\frac{1}{4 ! N}\left(\lambda_{0}+\frac{\eta_{0} \phi^{2}}{10 N}\right) \varphi^{4} \\
& -\left(\frac{12 \eta_{0} \phi_{a} \phi_{b}}{6 ! N^{2}}\right) \varphi_{a} \varphi_{b} \varphi^{2}-\frac{1}{5 !}\left(\frac{\eta_{0} \phi_{a}}{N^{2}} \varphi_{a} \varphi^{4}\right)-\frac{\eta_{0}}{6 ! N^{2}} \varphi^{6} .
\end{aligned}
$$

The effective action $\Gamma(\phi)$ is found by solving for $G_{a b}(x, y)$ the equation

$$
\frac{\delta \Gamma(\phi, G)}{\delta G_{a b}(x, y)}=0
$$

and substituting the solution in the generalized effective action $\Gamma(\phi, G)$. The vertices in the above equation contain factors of $1 / N$ or $1 / N^{2}$, but a $\varphi$ loop gives a factor of $N$ provided the $O(N)$ isospin flows around it alone and not into another part of the graph. We usually call such loops bubbles. Then at leading order in $1 / N$, the vacuum graphs are bubble trees with two or three bubbles at each vertex. The (2PI) graphs are shown in Fig. 1. It is straightforward to obtain 


$$
\Gamma_{2}(\phi, G)=\frac{-1}{4 ! N} \int d^{D} x\left(\lambda_{0}+\frac{\eta_{0} \phi^{2}}{10 N}\right)\left[G_{a a}(x, x)\right]^{2}-\frac{\eta_{0}}{6 ! N^{2}} \int d^{D} x\left[G_{a a}(x, x)\right]^{3} .
$$

Therefore Eq. (5) becomes

$$
\begin{aligned}
\frac{\delta \Gamma(\phi, G)}{\delta G_{a b}(x, y)}= & \frac{1}{2}\left(G^{-1}\right)_{a b}(x, y)+\frac{i}{2} D^{-1}(\phi)-\frac{1}{12 N}\left(\lambda_{0}+\frac{\eta_{0} \phi^{2}}{10 N}\right)\left[\delta_{a b} G_{c c}(x, x)\right] \delta^{D}(x-y) \\
& -\frac{3 \eta_{0}}{6 ! N} \delta_{a b}\left[G_{c c}(x, x)\right]^{2} \delta^{D}(x-y)=0 .
\end{aligned}
$$

Rewriting this equation, we obtain the gap equation

$$
\begin{aligned}
\left(G^{-1}\right)_{a b}(x, y)= & D_{a b}^{-1}(\phi ; x, y)+\frac{i}{6 N}\left(\lambda_{0}+\frac{\eta_{0} \phi^{2}}{10 N}\right)\left[\delta_{a b} G_{c c}(x, x)\right] \delta^{D}(x-y) \\
& +\frac{i \eta_{0}}{5 ! N^{2}} \delta_{a b}\left[G_{c c}(x, x)\right]^{2} \delta^{D}(x-y) .
\end{aligned}
$$

Hence

$$
\frac{i}{2} \operatorname{Tr} D^{-1} G=\frac{1}{12 N} \int d^{D} x\left(\lambda_{0}+\frac{\eta_{0} \phi^{2}}{10 N}\right)\left[G_{a a}(x, x)\right]^{2}+\frac{3 \eta_{0}}{6 ! N^{2}} \int d^{D} x\left[G_{a a}(x, x)\right]^{3}+c t e .
$$

Using Eqs. (8) and (9) in Eq. (6) we find the effective action

$$
\Gamma(\phi)=I(\phi)+\frac{i}{2} \operatorname{Tr}\left[\operatorname{Ln} G^{-1}\right]+\frac{1}{4 ! N} \int d^{D} x\left(\lambda_{0}+\frac{\eta_{0} \phi^{2}}{10 N}\right)\left[G_{a a}(x, x)\right]^{2}+\frac{2 \eta_{0}}{6 ! N^{2}} \int d^{D} x\left[G_{a a}(x, x)\right]^{3},
$$

where $G_{a b}$ is given implicitly by Eq. (8). The trace in (10) are both the functional and the internal space. The last two terms on the right-hand side of Eq. (10) are the leading contribution to the effective action in the $1 / N$ expansion. As usual we may simplify the situation by separating $G_{a b}$ into transverse and longitudinal components, so

$$
G_{a b}=\left(\delta_{a b}-\frac{\phi_{a} \phi_{b}}{\phi^{2}}\right) g+\frac{\phi_{a} \phi_{b}}{\phi^{2}} \widetilde{g},
$$

in this form we can invert $G_{a b}$,

$$
(G)_{a b}^{-1}=\left(\delta_{a b}-\frac{\phi_{a} \phi_{b}}{\phi^{2}}\right) g^{-1}+\frac{\phi_{a} \phi_{b}}{\phi^{2}} \widetilde{g}^{-1} .
$$

Now we can take the trace with respect to the indices of the internal space,

$$
G_{a a}=N g+O(1), \quad(G)_{a a}^{-1}=N g^{-1}+O(1) .
$$

From this equation at leading order in $1 / N, G_{a b}$ is diagonal in $a, b$. Substituting Eq. (13) into Eq. (10) and Eq. (8) and keeping only the leading order one finds that the daisy and superdaisy resumed effective potential for the $\varphi^{6}$ theory is given by

$$
\Gamma(\phi)=I(\phi)+\frac{i N}{2} \operatorname{tr}\left(\ln g^{-1}\right)+\frac{N}{4 !} \int d^{D} x\left(\lambda_{0}+\frac{\eta_{0} \phi^{2}}{10 N}\right) g^{2}(x, x)+\frac{2 N \eta_{0}}{6 !} \int d^{D} x g^{3}(x, x)+O(1),
$$

where the trace is only in the functional space, and the gap equation becomes 


$$
g^{-1}(x, y)=i\left[\square+m_{0}^{2}+\frac{\lambda_{0}}{6}\left(\frac{\phi^{2}}{N}+g(x, x)\right)+\frac{\eta_{0}}{5 !}\left(\frac{\phi^{2}}{N}+g(x, x)\right)^{2}\right] \delta^{D}(x-y)+O\left(\frac{1}{N}\right) .
$$

It is important to point out that this calculation was done by Townsend. ${ }^{5}$

\section{THE THEORY AT FINITE TEMPERATURE}

In order to generalize these results to the case of finite temperature we are going to assume that the system is in equilibrium with a thermal bath a temperature $T=\beta^{-1}$. Since we are interested in the equilibrium situation it is convenient to use the Matsubara formalism (imaginary time). Consequently it is convenient to continue all momenta to Euclidean values $\left(p_{0}=i p_{4}\right)$ and take the following Ansatz for $g(x, y)$ :

$$
g(x, y)=\int \frac{d^{D} p}{(2 \pi)^{D}} \frac{\exp ^{i(x-y) p}}{p^{2}+M^{2}(\phi)} .
$$

Substituting Eq. (16) in Eq. (15) we get the expression for the gap equation,

$$
M^{2}(\phi)=m_{0}^{2}+\frac{\lambda_{0}}{6}\left(\frac{\phi^{2}}{N}+F(\phi)\right)+\frac{\eta_{0}}{5 !}\left(\frac{\phi^{2}}{N}+F(\phi)\right)^{2},
$$

where $F(\phi)$ is given by

$$
F(\phi)=\int \frac{d^{D} p}{(2 \pi)^{D}} \frac{1}{p^{2}+M^{2}(\phi)},
$$

and the effective potential in the $D$-dimensional Euclidean space can be written as

$$
V(\phi)=V_{0}(\phi)+\frac{N}{2} \int \frac{d^{D} p}{(2 \pi)^{D}} \ln \left[p^{2}+M^{2}(\phi)\right]-\frac{N}{4 !}\left(\lambda_{0}+\frac{\eta_{0} \phi^{2}}{10 N}\right) F(\phi)^{2}-\frac{2 N \eta_{0} F(\phi)^{3}}{6 !}
$$

where $V_{0}(\phi)$ is the tree approximation of the potential.

Replacing the continuous four momenta $k_{4}$ by discrete $\omega_{n}$ and the integration by a summation $(\beta=1 / T)$. The effective potential at finite temperature is

$$
V_{\beta}(\phi)=V_{0}(\phi)+\frac{N}{2 \beta} \sum_{n}^{\infty} \int \frac{d^{D-1}}{(2 \pi)^{D-1}} \ln \left[\omega_{n}+p^{2}+M_{\beta}^{2}(\phi)\right]-\frac{N}{4 !}\left(\lambda_{0}+\frac{\eta_{0} \phi^{2}}{10 N}\right) F_{\beta}(\phi)^{2}-\frac{2 N \eta F_{\beta}(\phi)^{3}}{6 !}
$$

where the expression $F_{\beta}(\phi)$ is the finite temperature generalization of $F(\phi)$, and is given by

$$
F_{\beta}(\phi)=\frac{1}{\beta} \sum_{n=-\infty}^{\infty} \int \frac{d^{D-1} p}{(2 \pi)^{D-1}} \frac{1}{\omega_{n}^{2}+p^{2}+M_{\beta}^{2}(\phi)} .
$$

The gap equation for this theory at finite temperature is

$$
M_{\beta}^{2}(\phi)=m_{0}^{2}+\frac{\lambda_{0}}{6}\left(\frac{\phi^{2}}{N}+F_{\beta}(\phi)\right)+\frac{\eta_{0}}{5 !}\left(\frac{\phi^{2}}{N}+F_{\beta}(\phi)\right)^{2} .
$$

In order to regularize $F_{\beta}(\phi)$ given by Eq. (21), we use a mixing between dimensional regularization and analytic regularization. For this purpose we define the following expression: 


$$
I_{\beta}(D, s, m)=\frac{1}{\beta} \sum_{n=-\infty}^{\infty} \int \frac{d^{D-1} k}{(2 \pi)^{D-1}} \frac{1}{\left(\omega_{n}^{2}+k^{2}+m^{2}\right)^{s}} .
$$

The analytic extension of the inhomogeneous Epstein zeta function can be done and the corresponding analytic extension of $I_{\beta}(D, s, m)$ is

$$
I_{\beta}(D, s, m)=\frac{m^{D-2 s}}{\left(2 \pi^{1 / 2}\right)^{D} \Gamma(s)}\left[\Gamma\left(s-\frac{D}{2}\right)+4 \sum_{n=1}^{\infty}\left(\frac{2}{m n \beta}\right)^{D / 2-s} K_{D / 2-s}(m n \beta)\right],
$$

where $K_{\mu}(z)$ is the modified Bessel function of the third kind. Fortunately for $D=2+1$ the analytic extension of the function $I_{\beta}\left(D, s=1, m=M_{\beta}(\phi)\right)=F_{\beta}(\phi)$ is finite and can be expressed in a closed form $^{6}$

$$
F_{\beta}(\phi)=I_{\beta}\left(3,1, M_{\beta}(\phi)\right)=-\frac{M_{\beta}(\phi)}{4 \pi}\left(1+\frac{2 \ln \left(1-e^{-M_{\beta}(\phi) \beta}\right)}{M_{\beta}(\phi) \beta}\right) .
$$

We note that in $D=2+1$ we have no pole, at least in this approximation. To proceed to regularize the second term of Eq. (20), we define

$$
L F_{\beta}(\phi)=\frac{1}{\beta} \sum_{n=1}^{\infty} \int \frac{d^{D-1} p}{(2 \pi)^{D-1}} \ln \left[\omega_{n}+p^{2}+M_{\beta}^{2}(\phi)\right]
$$

then,

$$
\frac{\partial L F_{\beta}(\phi)}{\partial M_{\beta}}=\left(2 M_{\beta}\right) \frac{1}{\beta} \sum_{n=1}^{\infty} \int \frac{d^{D-1} p}{(2 \pi)^{D-1}} \frac{1}{\omega_{n}+p^{2}+M_{\beta}^{2}(\phi)}
$$

and from Eq. (21), we have that

$$
\frac{\partial L F_{\beta}(\phi)}{\partial M_{\beta}}=\left(2 M_{\beta}\right) F_{\beta}(\phi) .
$$

For $D=2+1, F_{\beta}(\phi)$ is finite and is given by Eq. (29) (Ref. 6) and integrating the Eq. (28), we obtain

$$
L F_{\beta}(\phi)_{R}=-\frac{M_{\beta}(\phi)^{3}}{6 \pi}-\frac{M_{\beta}(\phi) L i_{2}\left(e^{-M_{\beta}(\phi) \beta}\right)}{\pi \beta^{2}}-\frac{L i_{3}\left(e^{-M_{\beta}(\phi) \beta}\right)}{\pi \beta^{3}} .
$$

The definition of general polylogarithm function $L_{n}(z)$ can be found in Ref. 7 .

The daisy and super daisy resummed effective potential at finite temperature for $D=2+1$ is then given by

$$
V_{\beta}(\phi)=V_{0}(\phi)+\frac{N}{2} L F_{\beta}(\phi)_{R}-\frac{N}{4 !}\left(\lambda_{0}+\frac{\eta_{0} \phi^{2}}{10 N}\right)\left(F_{\beta}(\phi)_{R}\right)^{2}-\frac{2 N \eta\left(F_{\beta}(\phi)_{R}\right)^{2}}{6 !}
$$

and the corresponding gap equation [see Eq. (22)]

$$
\begin{aligned}
M_{\beta}^{2}(\phi)= & m_{0}^{2}+\frac{\lambda_{0}}{6}\left(\frac{\phi^{2}}{N}-\frac{M_{\beta}(\phi)}{4 \pi}\left[1+\frac{2 \ln \left(1-e^{-M_{\beta}(\phi) \beta}\right)}{M_{\beta}(\phi) \beta}\right]\right) \\
& +\frac{\eta_{0}}{5 !}\left(\frac{\phi^{2}}{N}-\frac{M_{\beta}(\phi)}{4 \pi}\left[1+\frac{2 \ln \left(1-e^{-M_{\beta}(\phi) \beta}\right)}{M_{\beta}(\phi) \beta}\right]\right)^{2} .
\end{aligned}
$$

From this expression we can deduce that there is no possible way to find a solution for $M_{\beta}$ going to zero, because the terms in Eq. (31) containing the logarithm will not permit, and this situation is similar to the scalar theory with $O(N)$ symmetry in two dimensions (2D) at zero temperature. ${ }^{8}$ 
This result is in agreement with the the Coleman-Mermin-Wagner theorem, ${ }^{9}$ which statement is related to the fact that it is impossible to construct a consistent theory of massless scalar in 2D. If a spontaneous breaking of continuous symmetry were to happen at finite $T$, then one would be faced with this problem at momentum scales below $T_{c}$, i.e., it would be impossible to construct an effective 2D theory of the Goldstone bosons zero modes.

\section{CONCLUSIONS}

In this paper we have found the daisy and super daisy effective potential for the theory $\varphi^{6}$ in $D=2+1$-dimensional Euclidean space at finite temperature. The form of effective potential have been found explicitly using resummation method in the leading order $1 / N$ approximation (Hartree-Fock approximation). We found that in this approximation there is no symmetry breaking for any temperature and this is clearly a manifestation of the Coleman-Mermin-Wagner theorem which stipulates that the spontaneous symmetry breaking of continuous symmetry cannot happen in $D=2+1$ at finite temperature.

\section{ACKNOWLEDGMENT}

This paper was supported by FAPESP.

${ }^{1}$ W. A. Barden, M. Moshe, and M. Bander, Phys. Rev. Lett. 52, 1118 (1984).

${ }^{2}$ L. Dolan and R. Jackiw, Phys. Rev. D 9, 3320 (1974).

${ }^{3}$ J. M. Cornwall, R. Jackiw, and E. Tomboulis, Phys. Rev. D 10, 2428 (1974).

${ }^{4}$ R. Jackiw, Diverses Topics in Theoretical and Mathematical Physics (World Scientific, Singapore, 1995).

${ }^{5}$ P. K. Townsend, Phys. Rev. D 12, 2269 (1975); Nucl. Phys. B 118, 199 (1977).

${ }^{6}$ G. N. J. Ananos and N. F. Svaiter, Physica A 241, 627 (1997).

${ }^{7}$ L. Lewin, Polylogarithms and Associated Functions (North-Holland, Amsterdam, 1981); http://mathworld.wolfram.com/ Polylogarithm.html

${ }^{8}$ S. R. Coleman, Phys. Rev. D 10, 2491 (1974).

${ }^{9}$ N. D. Mermin and H. Wagner, Phys. Rev. Lett. 17, 1133 (1966); S. R. Coleman, Commun. Math. Phys. 31, 259 (1973). 